\title{
La quimioprofilaxis antibiótica disminuyó la incidencia de malaria y anemia en niños de áreas endémicas
}

Chandramihan y col. BMJ.2005;331:727-733

\section{Objetivo}

Evaluar el efecto del tratamiento preventivo intermitente (TPI) en niños sobre la incidencia de malaria y anemia

\section{Diseño}

Ensayo clínico aleatorizado en grupos o "clusters*".

Lugar

Treinta centros de salud en Ghana, Africa.

\section{Pacientes}

Se estudiaron 2485 niños (placebo=1242; control=1243) pertenecientes a 96 hogares previamente aleatorizados en grupos de ocho.

\section{Intervención}

$\mathrm{Se}$ administraron dosis progresivas de sulfadoxina-pirimetamina (SP) y placebo durante el segundo y tercer refuerzo de la vacuna DPT y la primera dosis de antisarampionosa, más $15 \mathrm{mg} / \mathrm{día}$ de hierro durante el mes posterior a la vacunación. El seguimiento fue de 24 meses mediante visitas domiciliarias, cuestionarios de morbilidad, determinaciones de parasitemia, migraciones y certificados de defunción.

\section{Medición de Resultados principales}

Incidencia de malaria, anemia (hematocrito menor a 24\%) parasitemia (más de $5000 \mathrm{p} / \mu \mathrm{L}$ ) y admisiones hospitalarias por malaria. El análisis fue por intención de tratar, con estratificación por edad (2 a 15 y 16 a 24 meses) y estacionalidad; con ajuste multivariable* por sexo, residencia urbana/rural y uso de mosquitero.

\section{Resultados}

EI TPI hasta los 15 meses de edad se asoció a una disminución del riesgo de malaria, anemia, parasitemia y admisión hospitalaria. El efecto protector se mantuvo hasta los 24 meses. No se observaron beneficios del TPI en los niños de 16 a 24 meses. Ver tabla 1.

Tabla 1. Asociación entre haber recibido tratamiento preventivo y los resultados principales (efecto protector del tratamiento).

\begin{tabular}{c|c|c}
\multirow{2}{*}{ Desarrollo de malaria } & $\mathbf{2}$ OA $\mathbf{1 5}$ meses & $\mathbf{1 6}$ a 24 meses \\
\cline { 2 - 3 } & $0,75(0,66-0,86)$ & $1,05 \mathrm{NS}$ \\
\hline Presencia de parasitemia & $0,76(0,66-0,89)$ & $1,19 \mathrm{NS}$ \\
\hline Admisión hospitalaria por malaria & $0,60(0,42-0,86)$ & $0,92 \mathrm{NS}$ \\
\hline Anemia & $0,65(0,47-0,89)$ & $1,09 \mathrm{NS}$ \\
\hline Malaria y anemia & $0,47(0,28-, 77)$ & $1,28 \mathrm{NS}$ \\
\hline Muerte por malaria o anemia & $1,21 \mathrm{NS}$ & $1,17 \mathrm{NS}$ \\
\hline
\end{tabular}

NS: Diferencia no significativa.

\section{Conclusiones}

EL TPI disminuyó la incidencia de malaria y anemia en niños de dos a 15 meses. Existen dudas acerca de un posible efecto rebote en el segundo año de vida.

Fuente de financiamiento: Departament for International Development. Gates Malaria Partnership.

\section{Comentario}

Anualmente el paludismo afecta a unos 350 a 500 millones de personas de las que mueren un millón. En las áreas endémicas (sudeste de Asia, Africa, Sudamérica y Caribe) alcanza una prevalencia del $40 \%$, da cuenta del $50 \%$ de ingresos hospitalarios y del $20 \%$ de la mortalidad en menores de cinco años. También existe aumento de la resistencia a la monoterapia (cloroquina o sulfadoxina-pirmetamina) y una morbi-mortalidad incrementada en asociación al VIH-SIDA ${ }^{1-3}$.

OMS y UNICEF fijaron como meta que el $60 \%$ de la población endémica debe tener acceso al tratamiento preventivo: uso de mosquiteros con insecticidas, lucha anti-vectorial y rociamiento de viviendas ${ }^{4}$. Con respecto al TPI, actualmente se realiza solo en embarazadas, para disminuir la incidencia de bajo peso al nacer, prematurez, mortalidad neonatal y anemia.

El presente trabajo, está destinado a comprobar los beneficios del TPI con antibióticos sobre indicadores de malaria en una población infantil africana con factores ambientales y sociales similares a los de algunas regiones de Sudamérica y aunque fue limitado en cuanto al grupo etareo, estudiando sólo a los menores de dos años, es destacable por la adecuada metodología y el plan de seguimiento comunitario implementado acorde a las múltiples variables tenidas en cuenta.

Con respecto a la disminución de incidencia de anemia y malaria, confirma los resultados obtenidos por estudios anteriores, agregándose el menor ingreso hospitalario y el mayor efecto protector con la aplicación estacional (lluviosa- seca) de la dosis sin obtenerse aún beneficios con respecto a la mortalidad infantil. Resulta interesante el seguimiento a largo plazo en cuanto a la profilaxis antibiótica, no solo sobre el impacto logrado en la incidencia sino también en poder disminuir la mortalidad por malaria. Hasta el momento, el uso de mosquiteros tratados con insecticidas es el método que demostró reducir significativamente un $50 \%$ la incidencia de malaria en menores de cinco años de áreas endémicas 5 .

\section{Conclusiones del comentador}

El presente estudio aporta datos concluyentes sobre la ventaja de implementar TPI en la población infantil de riesgo para reducir la incidencia y la morbilidad por malaria.

Gloria Vega [ Médica Especialista en Medicina Familiar. Instituto de Obras Sociales del Ejército. Provincia de Salta.]

Vega G. La quimioprofilaxis antibiótica disminuyó la incidencia de malaria y anemia en niños de áreas endémicas. Evid. actual. práct. ambul. 9(3);69. May-Jun 2006. Comentado de: Chandramihan, D; Owusu-Agyei, S; Carneiro I. Cluster randomised trial of intermittent preventive treatment for malaria in infants in area of high seasonal tranmission in Ghana. BMJ.2005;331:727-733. PMID: 16195288

\section{Referencias}

1. Snow R, Guerra C, Noor A, Myint H, Hay S. The global distribution of clinical episodes of Plasmodium falciparum malaria. Nature 2005;434: 214-7.

2. Murray CJ, Lopez AD. Global burden of disease and injury series. Geneva: World Health Organization, 1996: 320-7.

3. Murphy SC, Breman JG. Gaps in the childhood malaria burden in Africa: cerebral malaria, neurological sequelae, anaemia, respiratory distress, hypogly-

caemia and complications of pregnancy. Am J Trop Med Hyg 2001;64(1-2 suppl): 57-67.

4. Sachs JD. Achieving the Millennium Development Goals--the case of malaria. N Engl J Med. 2005;352(2):115-7.

5. Lengeler C. Insecticide-treated bed nets and curtains for preventing malaria. Cochrane Database Syst Rev 2004;(2): CD000363. 